\title{
Practical Use of Gemcitabine and Cisplatin Combination Therapy as First-Line Treatment for Japanese Patients with Advanced Biliary Tract Cancer*
}

\author{
Hisato Kawakami ${ }^{1}$, Isamu Okamoto, ${ }^{1,2 \#}$, Wataru Okamoto ${ }^{1}$, Masayuki Takeda ${ }^{1}$, Shinya Ueda ${ }^{1}$, \\ Toshihiro Kudo ${ }^{1}$, Shin-ichi Nishina ${ }^{1}$, Yasuhito Fujisaka ${ }^{1}$, Masaki Miyazaki ${ }^{1}$, Junji Tsurutani ${ }^{1}$, \\ Takayasu Kurata ${ }^{1}$, Kazuhiko Nakagawa ${ }^{1}$
}

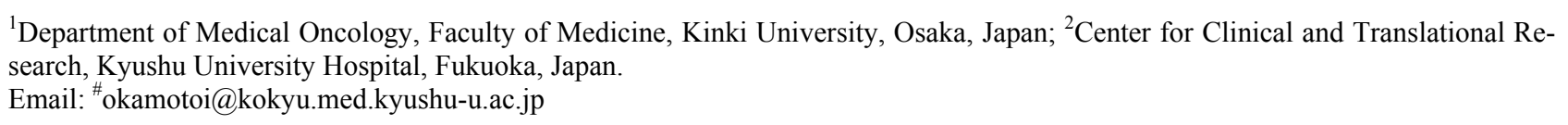

Received May $14^{\text {th }}, 2013$; revised June $16^{\text {th }}, 2013$; accepted June $23^{\text {rd }}, 2013$

Copyright (C) 2013 Hisato Kawakami et al. This is an open access article distributed under the Creative Commons Attribution License, which permits unrestricted use, distribution, and reproduction in any medium, provided the original work is properly cited.

\begin{abstract}
Gemcitabine and cisplatin combination therapy (GC) is accepted as a standard treatment for advanced biliary tract cancer (BTC). However, little information is available regarding such treatment in the clinical practice setting in Japan. We retrospectively examined the clinical data of patients with unresectable or recurrent BTC who received GC as first-line treatment. The regimen consisted of cisplatin $\left(25 \mathrm{mg} / \mathrm{m}^{2}\right)$ and gemcitabine $\left(1000 \mathrm{mg} / \mathrm{m}^{2}\right)$ administered intravenously on days 1 and 8 of repeated 3-week cycles. Twenty patients were analyzed. A total of 148 cycles of GC was administered, with a median of 8 and a range of 1 to 18 cycles. Treatment delay and dose reduction were noted in $35(24 \%)$ and 41 (28\%) of the 148 cycles, respectively. The major adverse events of grade 3 or 4 included neutropenia $(50 \%)$, leukopenia $(45 \%)$, anemia $(30 \%)$, and thrombocytopenia $(15 \%)$. Nonhematologic toxicities included nausea $(10 \%)$, appetite loss $(10 \%)$, and fatigue $(10 \%)$. Median progression-free and overall survival times were 6.9 and 12.3 months, respectively. Gallbladder cancer showed a significantly higher response rate than did other types of BTC (chi-squaretest, $P=0.002$ ). GC was thus effective and well tolerated as first-line chemotherapy for Japanese patients with advanced BTC in the clinical practice setting.
\end{abstract}

Keywords: Gemcitabine; Cisplatin; Chemotherapy; Biliary Tract Cancer

\section{Introduction}

Biliary tract cancer (BTC) is a rare type of cancer worldwide, but it is more common in East Asia and Latin America than in other regions [1]. In Japan, BTC is the sixth leading cause of death from cancer [2] and its prevalence is increasing. Although the most effective treatment for localized disease is surgery, most cases of BTC are diagnosed as advanced and inoperable, despite substantial progress in diagnostic imaging. Outcomes are extremely poor in such patients, with a median survival time of 2.5 months with best supportive care [3].

Gemcitabine has shown antitumor activity in patients with BTC, as revealed by the results of predominantly phase II studies [4-6], and this drug is generally used in

${ }^{*}$ Conflict of interest statement: None declared.

\#Corresponding author. the palliative setting, yielding a median survival time of 6 to 9 months. Cisplatin is a key anticancer agent for solid tumors and is widely administered in combination chemotherapy. Gemcitabine and cisplatin combination therapy (GC) has shown promising antitumor efficacy in several phase II studies with BTC patients [7-12]. Given these results, a phase III trial comparing GC with gemcitabine alone was conductedfor locally advanced or metastatic BTC in the United Kingdom (ABC-02 study). A total of 410 patients were randomly assigned to receive gemcitabine $\left(1000 \mathrm{mg} / \mathrm{m}^{2}\right.$ on days 1,8 , and 15 of a 4week cycle) or GC $\left(1000 \mathrm{mg} / \mathrm{m}^{2}\right.$ and $25 \mathrm{mg} / \mathrm{m}^{2}$, respectively, on days 1 and 8 of a 3 -week cycle). The median overall survival (OS)was significantly better forthe patients receiving $\mathrm{GC}$ than for those receiving gemcitabine alone (11.7 versus 8.1 months; hazard ratio [HR] of 0.64 , with a $95 \%$ confidence interval $[\mathrm{CI}]$ of 0.52 to $0.80 ; P<$ 
0.001).The median progression-free survival (PFS) was also significantly longer for the GC group than for the gemcitabine group (8.0 versus 5.0 months; HR of 0.63 , with a $95 \% \mathrm{CI}$ of 0.51 to $0.77 ; P<0.001$ ) [13]. On the basis of the results of the $\mathrm{ABC}-02$ study, $\mathrm{GC}$ was recognized as the standard of care for the treatment of advanced BTC.A randomized phase II study comparing GC with gemcitabine alone was also performed for locally advanced or metastatic BTC in Japan (BT22 study), with the same treatment dose and scheduleas adopted in the ABC-02 study. Overall, 84 patients were randomized to receive either GC or gemcitabine alone. The 1-year survival rate, which was the primary endpoint of the study, was higher in the GC group than in the gemcitabine group (39.0 versus $31.9 \%$ ) [14]. The findings of the $\mathrm{ABC}$ 02 and BT22 studies have thus resulted in GC becoming accepted as a standard treatment for patients with BTC in Japan. To date, however, information regarding the safety and efficacy of GC in Japanese individuals with BTC has been limited to that obtained from 42 patients in the BT 22 study. The safety and efficacy of GC in the clinical practice setting have thus remained uncertain. We now report our experience with GC for Japanese patients with BTC in the clinical practice setting.

\section{Methods}

\subsection{Eligibility Criteria}

We reviewed the cases in our database and retrospectively examined the clinical data of patients with unresectable or recurrent BTC who received GC as the firstline treatment. Patients were eligible if they had: 1) pathologically or radiographically confirmed BTC;2) an Eastern Cooperative Oncology Group (ECOG) performance status of 0 to 2 ; and 3 ) adequate bone marrow function (white blood cell count of $>3000 / \mathrm{mm}^{3}$, hemoglobin content of $>9.0 \mathrm{~g} / \mathrm{dl}$, and a platelet count of $>100,000$ / $\mathrm{mm}^{3}$ ), liver function (total serum bilirubin concentration of less than three times the upper limit of normal [ULN], and serum aspartate and alanine transaminase levels of less than five times the ULN), and renal function (serum creatinine concentration of $<1.2 \mathrm{mg} / \mathrm{dl}$ and creatinine clearance of $>50 \mathrm{ml} / \mathrm{min}$ ). In patients with obstructive jaundice, the total serum bilirubin concentration was required to be less than three times the ULN after biliary drainage. Written informed consent was obtained from each patient prior to treatment administration.

\subsection{Treatment Schedule}

GC was administered mostly on an outpatient basis. Gemcitabine was given intravenously $\left(1000 \mathrm{mg} / \mathrm{m}^{2}\right)$ over $30 \mathrm{~min}$ and cisplatin was administered intravenously $(25$ $\mathrm{mg} / \mathrm{m}^{2}$ ) over $120 \mathrm{~min}$ on days 1 and 8 of a 3 -week cycle.
Treatment was continued until disease progression, the occurrence of unacceptable toxicity, or patient refusal. We adopted the following general administration criteria for GC: a neutrophil count of $\geq 1500 / \mathrm{mm}^{3}$, a platelet count of $\geq 75,000 / \mathrm{mm}^{3}$, a serum total bilirubin concentration of $\leq 2.5 \mathrm{mg} / \mathrm{dl}$, a serum creatinine level of $\leq 1.5 \mathrm{mg} / \mathrm{dl}$, and other nonhematologic toxicity of grade 1 or less. Administration of gemcitabine alone after discontinuation of GC was allowed at the discretion of the physician, whereas administration of cisplatin alone was not allowed. Antiemetic prophylaxis with $5-\mathrm{HT}_{3}$ serotonin receptor antagonists plus dexamethasone was administered in all cases. A neurokinin-1 receptor antagonist was used at the physician's discretion.

\subsection{Toxicity Evaluation}

All adverse events were reviewed based on medical records and evaluated according to the National Cancer Institute Common Terminology Criteria for Adverse Events, version 4.0. The highest toxicity grade for each patient in all cycles of chemotherapy was used for toxicity analysis.

\subsection{Efficacy Measures}

The efficacy end points were tumor response, PFS, and OS. Tumor assessment by computed tomography of the abdomen and chest was performed at baseline and after two cycles of chemotherapy according to the Response Evaluation Criteria in Solid Tumors (RECIST) version 1.1. PFS was defined as the time from enrollment to the date of confirmation of progressive disease or of death from any cause, whichever occurred first. OS was defined as the time from registration until death from any cause. Patients not known to have died or to have developed progressive disease were censored at the date of the last progression-free assessment.

\subsection{Statistical Analysis}

Survival curves were constructed by the Kaplan-Meier method and were compared with the log-rank test. Differences in tumor response were evaluated with the chisquare test. Statistical analysis was performed with the use of IBM SPSS statistics software version 20. A $P$ value of $<0.05$ was considered statistically significant.

\section{Results}

\subsection{Patient Characteristics}

The characteristics of the 20 enrolled patients are listed in Table 1. The median age of the patients was 64.5 years, with similar numbers of men and women. Six individuals were 70 years of age or older. Five patients 
Table 1. Patient characteristics.

\begin{tabular}{|c|c|}
\hline Characteristic & No. of patients (\%) \\
\hline \multicolumn{2}{|l|}{ Sex } \\
\hline Male & $11(55 \%)$ \\
\hline Female & $9(45 \%)$ \\
\hline \multicolumn{2}{|l|}{ Age (years) } \\
\hline Median & 64.5 \\
\hline Range & $44-76$ \\
\hline \multicolumn{2}{|l|}{ Performance Status } \\
\hline $0 / 1 / 2$ & $7 / 11 / 2(35 \% / 55 \% / 10 \%)$ \\
\hline \multicolumn{2}{|l|}{ Primary tumor site } \\
\hline Extrahepatic bile duct & $6(30 \%)$ \\
\hline Intrahepatic bile duct & $8(40 \%)$ \\
\hline Gallbladder & $6(30 \%)$ \\
\hline \multicolumn{2}{|l|}{ Metastatic sites } \\
\hline Regional lymph nodes & $17(85 \%)$ \\
\hline Distant lymph nodes & $13(65 \%)$ \\
\hline Liver & $15(75 \%)$ \\
\hline Peritoneum & $2(10 \%)$ \\
\hline Lung & $2(10 \%)$ \\
\hline Other & $4(20 \%)$ \\
\hline \multicolumn{2}{|l|}{ Initial onset or recurrence } \\
\hline Initial onset & $15(75 \%)$ \\
\hline Recurrence after surgery & $5(25 \%)$ \\
\hline \multicolumn{2}{|l|}{ Histological type } \\
\hline Adenocarcinoma & $14(70 \%)$ \\
\hline Adenosquamous carcinoma & $1(5 \%)$ \\
\hline Cholangiocarcinoma & $1(5 \%)$ \\
\hline Not obtainable & $4(20 \%)$ \\
\hline \multicolumn{2}{|c|}{ Disease stage (extrahepatic bile duct cancer, gallbladder cancer) } \\
\hline IIA & $0(0 \%)$ \\
\hline IIB & $1^{\mathrm{a}}(5 \%)$ \\
\hline III & $1(5 \%)$ \\
\hline IV & $6(30 \%)$ \\
\hline Reccurence after surgery & $4(20 \%)$ \\
\hline \multicolumn{2}{|c|}{ Disease stage (intrahepatic bile duct cancer) } \\
\hline II & $1^{\mathrm{a}}(5 \%)$ \\
\hline III & $0(0 \%)$ \\
\hline IVA & $2(10 \%)$ \\
\hline IVB & $4(20 \%)$ \\
\hline Reccurence after surgery & $1(5 \%)$ \\
\hline \multicolumn{2}{|l|}{ Biliary drainage } \\
\hline Yes & $2(10 \%)$ \\
\hline No & $18(90 \%)$ \\
\hline
\end{tabular}

a Patients were diagnosed as having unresectable disease with marked regional node metastases involving the proper hepatic artery or main portal vein.
(25\%) had recurrent metastatic disease after surgical resection, and $15(75 \%)$ had unresectable metastatic disease at the initial diagnosis. Tumor specimens were obtained from 16 individuals, including 14 patients with adenocarcinoma, one patient with adenosquamous carcinoma, and one patient with cholangiocarcinoma. The primary tumor sites included the gallbladder in six patients $(30 \%)$, the intrahepatic bile duct in eight patients (40\%), and the extrahepatic bile duct in six patients $(30 \%)$. Regional lymph nodes were the most common metastatic site, followed by the liver and distant lymph nodes.

\subsection{Treatment Delivery}

The data for treatment delivery are summarized in Table 2. Seventeen patients $(85 \%)$ required a treatment delay and eight patients $(40 \%)$ required dose reduction. A total of 148 cycles of GC was administered, with a median of 8 and a range of 1 to 18 cycles per patient. Treatment delays and dose reductions were noted in $35(24 \%)$ and $41(28 \%)$ of the 148 cycles, respectively. Most treatment delays ( 24 out of 35 cycles) were due to hematologictoxicity that persisted for up to 7 days; the remaining $11 \mathrm{cy}$ cles were delayed for $>2$ weeks (15 to 70 days) be

Table 2. Summary of treatment delivery.

\begin{tabular}{|c|c|}
\hline Total treatment cycles & 148 \\
\hline Median no. of cycles (range) & $8(1-18)$ \\
\hline \multicolumn{2}{|l|}{ Treatment delay } \\
\hline Cycles (\%) & $35(24 \%)$ \\
\hline \multirow[t]{8}{*}{ Reasons (cycles) } & Neutropenia (19) \\
\hline & Fever (5) \\
\hline & Fatigue (3) \\
\hline & Anemia (2) \\
\hline & Patient's request (2) \\
\hline & Platelet count decreased (2) \\
\hline & $\begin{array}{l}\text { Serum creatinine } \\
\text { level increased (1) }\end{array}$ \\
\hline & Febrile neutropenia (1) \\
\hline \multicolumn{2}{|l|}{ Dose reduction } \\
\hline Cycles (\%) & $41(28 \%)$ \\
\hline \multirow[t]{4}{*}{ Reasons (cycles) } & Neutropenia (25) \\
\hline & Platelet count decreased (8) \\
\hline & Fatigue (4) \\
\hline & Febrile neutropenia (4) \\
\hline
\end{tabular}


cause of the development of prolonged neutropenia (6 cycles), prolonged thrombocytopenia (2 cycles), prolonged anemia with refusal of blood transfusion ( 1 cycle), febrile neutropenia of grade 3 ( 1 cycle), or fever of grade 1 ( 1 cycle). The reasons for dose reduction included the development ofneutropenia ( 25 of 41 cycles), thrombocytopenia ( 8 cycles), fatigue (4 cycles), or febrile neutropenia (4 cycles).Reasons for discontinuation of treatment included radiologically determined progressive disease (15 cases), treatment refusal ( 2 cases), and surgery with curative intent (1 case).

\subsection{Toxicity}

Major adverse events during the entire period are presented in Table 3. No treatment-related deaths occurred. The major adverse events of grade 3 or 4 included neutropenia $(50 \%)$, leukopenia $(45 \%)$, anemia $(30 \%)$, and thrombocytopenia (15\%). Although neutropenia was the most common hematologic toxicity, febrile neutropenia of grade 3 was observed in only one case $(5 \%)$. With regard to nonhematologic toxicity, no toxicities of grade 4 were observed and those of grade 3 included nausea $(10 \%)$, appetite loss $(10 \%)$, and fatigue $(10 \%)$, all of which were manageable. Biliary tract infection of grade 3 was seen in one patient $(5 \%)$, but it resolved within a

Table 3. Treatment-related toxicities (graded according to National Cancer Institute Common Terminology Criteria for Adverse Events) in the 20 study subjects.

\begin{tabular}{|c|c|c|c|c|}
\hline \multirow[b]{2}{*}{ Toxicity } & \multicolumn{4}{|c|}{ No. of patients $(n=20)$} \\
\hline & Grade 1 & Grade 2 & Grade 3 & Grade 4 \\
\hline Leukopenia & 1 & 1 & 8 & 1 \\
\hline Neutropenia & 1 & 0 & 6 & 4 \\
\hline Febrile neutropenia & - & - & 1 & 0 \\
\hline Thrombocytopenia & 8 & 4 & 2 & 1 \\
\hline Anemia & 5 & 6 & 6 & 0 \\
\hline $\begin{array}{l}\text { Serum creatinine } \\
\text { increased }\end{array}$ & 2 & 0 & 0 & 0 \\
\hline Constipation & 12 & 4 & 0 & 0 \\
\hline Nausea & 1 & 0 & 2 & 0 \\
\hline Appetite loss & 8 & 4 & 2 & 0 \\
\hline Fatigue & 7 & 4 & 2 & - \\
\hline Biliary tract infection & - & - & 1 & 0 \\
\hline Vomiting & 2 & 1 & 0 & 0 \\
\hline Fever & 3 & 0 & 0 & 0 \\
\hline Stomatitis & 3 & 0 & 0 & 0 \\
\hline $\begin{array}{l}\text { Peripheral sensory } \\
\text { neuropathy }\end{array}$ & 2 & 0 & 0 & 0 \\
\hline
\end{tabular}

week of antibiotic therapy.

\subsection{Response}

The chemotherapeutic responses are summarized in Table 4. All patients but one were assessable for tumor response. Although no individual achieved a complete response, six patients achieved a partial response, giving a best overall response rate of $30 \%$ (95\% CI, 15 to $52 \%$ ). Ten patients $(50 \%)$ showed stable disease, and the remaining three patients $(15 \%)$ had progressive disease. Five of the six responders had gallbladder cancer (GBC). Response differed significantly (chi-square test, $P=$ $0.002)$ between patients with GBC $(n=6)$ and those with other types of BTC $(n=14)$.

\subsection{Survival}

At the time of analysis, 18 patients had died of their disease. With a median potential follow-up time of 12.5 months, median OS and median PFS were 12.3 months and 6.9 months, respectively (Figure 1). Neither median OS nor median PFS differed significantly between patients with GBC and those with other forms of BTC.

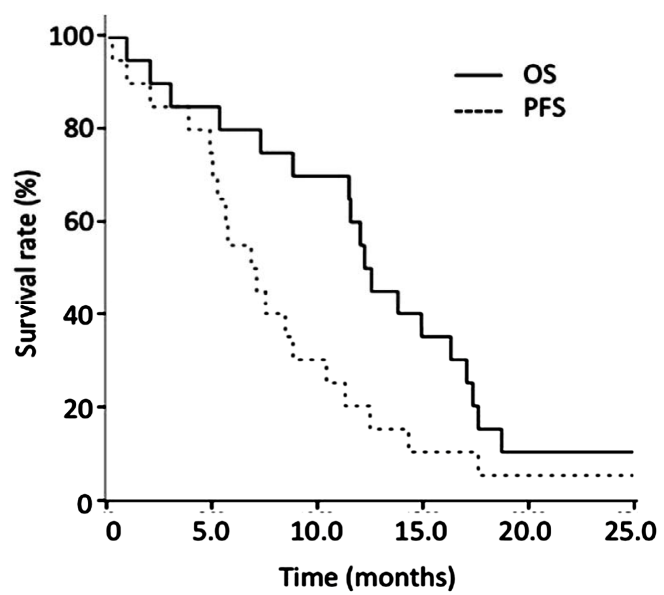

Figure 1. Kaplan-Meier analysis of OS and PFS for all patients $(n=20)$ from the onset of chemotherapy.

Table 4. Chemotherapeutic response according to tumor site.

\begin{tabular}{cccccc}
\hline \multirow{2}{*}{ Tumor site } & \multicolumn{5}{c}{ No. of patients } \\
\cline { 2 - 6 } & CR & PR & SD & PD & NE \\
\hline GBC & 0 & $5^{*}$ & 1 & 0 & 0 \\
Other BTCs & 0 & 1 & 9 & 3 & 1 \\
All (\%) & $0(0 \%)$ & $6(30 \%)$ & $10(50 \%)$ & $3(15 \%)$ & $1(5 \%)$ \\
\hline
\end{tabular}

Abbreviations: $\mathrm{CR}=$ complete response, $\mathrm{PR}=$ partial response, $\mathrm{SD}=$ stable disease, $\mathrm{PD}=$ progressive disease, $\mathrm{NE}=$ not evaluable, $\mathrm{GBC}=$ gall bladder cancer, BTC $=$ biliary tract cancer. ${ }^{*} P=0.002$ versus the corresponding value for other BTCs (chi-square test). 
Second-line chemotherapy was administered to 12 patients $(60 \%)$, all of whomreceived S-1 monotherapy.

\section{Discussion}

$\mathrm{GC}$ is now accepted worldwide as a standard regimen for first-line chemotherapy in patients with advanced BTC, largely on the basis of the results of the first large phase III study (ABC-02) showing the superiority of GC compared with gemcitabine monotherapy for this condition [13]. Although gemcitabine combined with oxaliplatin or capecitabine has shown promising efficacy for patients with BTC in single-arm phase II trials, yielding a response rate of $\sim 50 \%$ and OS of $\sim 14.0$ months [15], these treatments have not been evaluated in randomized phase III trials in comparison with gemcitabine alone. The safety and efficacy of GC for Japanese patients with BTC were also recently demonstrated in a randomized phase II trial (BT22) [14].GC was thus approved in February 2012 for the treatment of advanced BTC in Japan. Given the widespread adoption of GC for the treatment of advanced BTC, further information on its toxicity and treatment delivery characteristics in the clinical practice setting is of value.

Most toxicities observed in our study were hematologic in nature. The incidence ofleukopenia $(45 \%)$ or neutropenia $(50 \%)$ of grade 3 or 4 wassimilar to that observed in theprevious Japanese BT22 study (29.3 and $56.1 \%$, respectively) [14] but washigher than that apparent among Caucasiansin the ABC-02 study (15.7 and $25.3 \%$, respectively) [13], consistent with the notion of anethnic difference in the hematologic toxicity of chemotherapy between Japanese and Caucasian patients with BTC [16]. We further investigated the effect of dose reduction on efficacy. Overall, $40 \%(8 / 20)$ of patients required a dose reduction, mostly as a result of hematologic toxicity. Among the patients who underwent a dose reduction, the median PFS and response rate were 5.8 months and $12.5 \%$ ( 1 out of 8 patients), respectively. In contrast, a median PFS of 8.5 months and response rate of $41.7 \%$ (5 out of 12 patients) were apparent for the individuals who received the starting dose of GC throughout the treatment period. Nonhematologic toxicity was acceptable in the present study, with frequent adverse events including fatigue and gastrointestinal manifestations, both of which were clinically reversible. The overall profile and frequency of nonhematologic toxicities in our analysis are consistent with those observed in previous trials $[13,14]$.

Cisplatin is one of the most effective chemotherapeutic agents for the treatment of many types of solid tumors, but its administration is limited over the long term because of its cumulative toxicity, including neurological toxicity. Such problems occur even if cisplatin is admin- istered at a low dose. In the ABC-02 study, GC was delivered for up to a maximum of eight cycles, corresponding to a total cisplatin dose of $400 \mathrm{mg} / \mathrm{m}^{2}$ [13]. GC was continued for up to a maximum of 16 cycles in the BT22 study [14]. However, little has been known of the safety or efficacy of GC in patients receiving a total cumulative dose of cisplatin of $>400 \mathrm{mg} / \mathrm{m}^{2}$. In the present study, cisplatin was administered at a median total dose of $347.5 \mathrm{mg} / \mathrm{m}^{2}$, with a range of 25 to $550 \mathrm{mg} / \mathrm{m}^{2}$. Four of the 20 patients received cisplatin at $>400 \mathrm{mg} / \mathrm{m}^{2}$, and these individuals did not experience significant toxicity other than peripheral neuropathy of grade 1. Further studies are needed, however, to determine the optimal total dosage of cisplatin for treatment of BTC with GC.

Although patients with GBC showed a significantly higher response rate compared with those with other types of BTC in the present study, this finding is not particular to GC. Subgroup analysis of the ABC-02 study revealed a higher response rate for GBC than for other forms of BTC in both the gemcitabine arm (21.4 versus $11.7 \%)$ and the GC arm (37.7 versus $18.0 \%$ ) [13]. Furthermore, a previous pooled analysis of clinical trials revealed that GBC showed a higher response rate to drugs such as fluoropyrimidines, gemcitabine, and platinum compounds administered as single agents or in combination therapy [17]. These findings may indicate that BTC comprises a heterogeneous group of carcinomas that can be classified crudely as GBC or others. Indeed, recent studies have suggested that GBC and other forms of BTC should be considered as distinct diseases with different clinicopathologic characteristics [18-20].

In summary, our results suggest that GC is effective and well tolerated in Japanese patients with advanced BTC even in the clinical practice setting. Most toxicities observed in our study were hematologic, with such toxicities being a major cause of both dose reduction and treatment delay. Such characterization of GC is important for the optimal treatment of patients with BTC in clinical practice.

\section{REFERENCES}

[1] G. Randi, M. Malvezzi, F. Levi, J. Ferlay, E. Negri, S. Franceschi and C. La Vecchia, "Epidemiology of Biliary Tract Cancers: An Update," Annals of Oncology, Vol. 20, No. 1, 2009, pp. 146-159. doi:10.1093/annonc/mdn533

[2] (FPCR) FFPOCR, "Cancer Statistics Update," Number of Deaths (2008-2009), Cancer Site, 2010. http://www.fpcr.or.jp/pdf/statistics/fig01.pdf

[3] B. Glimelius, K. Hoffman, P. O. Sjoden, G. Jacobsson, H. Sellstrom, L. K. Enander, T. Linne and C. Svensson, "Chemotherapy Improves Survival and Quality of Life in Advanced Pancreatic and Biliary Cancer," Annals of Oncology, Vol. 7, No. 6, 1996, pp. 593-600. 
doi:10.1093/oxfordjournals.annonc.a010676

[4] T. Okusaka, H. Ishii, A. Funakoshi, K. Yamao, S. Ohkawa, S. Saito, H. Saito and T. Tsuyuguchi, "Phase II Study of Single-Agent Gemcitabine in Patients with Advanced Biliary Tract Cancer," Cancer Chemotherapy and Pharmacology, Vol. 57, No. 5, 2006, pp. 647-653. doi:10.1007/s00280-005-0095-3

[5] S. Kubicka, K. L. Rudolph, M. K. Tietze, M. Lorenz and M. Manns, "Phase II Study of Systemic Gemcitabine Chemotherapy for Advanced Unresectable Hepatobiliary Carcinomas," Hepato Gastroenterology, Vol. 48, No. 38, 2001, pp. 783-789.

[6] M. H. Lin, J. S. Chen, H. H. Chen and W. C. Su, "A Phase II Trial of Gemcitabine in the Treatment of Advanced Bile Duct and Periampullary Carcinomas," Chemotherapy, Vol. 49, No. 3, 2003, pp. 154-158. doi: $10.1159 / 000070622$

[7] S. T. Kim, J. O. Park, J. Lee, K. T. Lee, J. K. Lee, S. H. Choi, J. S. Heo, Y. S. Park, W. K. Kang and K. Park, "A Phase II Study of Gemcitabine and Cisplatin in Advanced Biliary Tract Cancer," Cancer, Vol. 106, No. 6, 2006, pp. 1339-1346. doi:10.1002/cncr.21741

[8] B. K. Park, Y. J. Kim, J. Y. Park, S. Bang, S. W. Park, J. B. Chung, K. S. Kim, J. S. Choi, W. J. Lee and S. Y. Song, "Phase II Study of Gemcitabine and Cisplatin in Advanced Biliary Tract Cancer," Journal of Gastroenterology and Hepatology, Vol. 21, No. 6, 2006, pp. 999-1003. doi:10.1111/j.1440-1746.2006.04230.x

[9] S. Thongprasert, S. Napapan, C. Charoentum and S. Moonprakan, "Phase II Study of Gemcitabine and Cisplatin as First-Line Chemotherapy in Inoperable Biliary Tract Carcinoma," Annals of Oncology, Vol. 16, No. 2, 2005, pp. 279-281. doi:10.1093/annonc/mdi046

[10] D. C. Doval, J. S. Sekhon, S. K. Gupta, J. Fuloria, V. K. Shukla, S. Gupta and B. S. Awasthy, "A Phase II Study of Gemcitabine and Cisplatin in Chemotherapy-Naive, Unresectable Gall Bladder Cancer," British Journal of Cancer, Vol. 90, No. 8, 2004, pp. 1516-1520. doi:10.1038/sj.bjc.6601736

[11] F. Giuliani, V. Gebbia, E. Maiello, N. Borsellino, E. Bajardi and G. Colucci, "Gemcitabine and Cisplatin for Inoperable and/or Metastatic Biliary Tree Carcinomas: A Multicenter Phase II Study of the Gruppo Oncologico dell'Italia Meridionale (GOIM)," Annals of Oncology, Vol. 17, No. 7, 2006, pp. 73-77. doi:10.1093/annonc/mdl956

[12] J. W. Valle, H. Wasan, P. Johnson, E. Jones, L. Dixon, R. Swindell, S. Baka, A. Maraveyas, P. Corrie, S. Falk, S. Gollins, F. Lofts, L. Evans, T. Meyer, A. Anthoney, T. Iveson, M. Highley, R. Osborne and J. Bridgewater, "Gemcitabine alone or in Combination with Cisplatin in Patients with Advanced or Metastatic Cholangiocarcinomas or Other Biliary Tract Tumours: A Multicentre Randomised Phase II Study: The UK ABC-01 Study," British Journal of Cancer, Vol. 101, No. 4, 2009, pp. 621-627. doi:10.1038/sj.bjc.6605211

[13] J. Valle, H. Wasan, D. H. Palmer, D. Cunningham, A. Anthoney, A. Maraveyas, S. Madhusudan, T. Iveson, S. Hughes, S. P. Pereira, M. Roughton and J. Bridgewater, "Cisplatin plus Gemcitabine versus Gemcitabine for Biliary Tract Cancer," New England Journal of Medicine, Vol. 362, No. 14, 2010, pp. 1273-1281. doi:10.1056/NEJMoa0908721

[14] T. Okusaka, K. Nakachi, A. Fukutomi, N. Mizuno, S. Ohkawa, A. Funakoshi, M. Nagino, S. Kondo, S. Nagaoka, J. Funai, M. Koshiji, Y. Nambu, J. Furuse, M. Miyazaki and Y. Nimura, "Gemcitabine alone or in Combination with Cisplatin in Patients with Biliary Tract Cancer: A Comparative Multicentre Study in Japan," British Journal of Cancer, Vol. 103, No. 4, 2010, pp. 469-474. doi:10.1038/sj.bjc. 6605779

[15] D. Marino, F. Leone, G. Cavalloni, C. Cagnazzo and M. Aglietta, "Biliary Tract Carcinomas: From Chemotherapy to Targeted Therapy," Critical Reviews in Oncology/Hematology, Vol. 85, No. 2, 2013, pp. 136-148. doi:10.1016/j.critrevonc.2012.06.006

[16] V. H. Phan, M. M. Moore, A. J. McLachlan, M. PiquetteMiller, H. Xu and S. J. Clarke, "Ethnic Differences in Drug Metabolism and Toxicity from Chemotherapy," Expert Opinion on Drug Metabolism \& Toxicology, Vol. 5, No. 3, 2009, pp. 243-257. doi:10.1517/17425250902800153

[17] F. Eckel and R. M. Schmid, "Chemotherapy in Advanced Biliary Tract Carcinoma: A Pooled Analysis of Clinical Trials," British Journal of Cancer, Vol. 96, 2007, pp. 896-902. doi:10.1038/sj.bjc.6603648

[18] H. S. Won, M. A. Lee, E. S. Chung, D. G. Kim, Y. K. You, T. H. Hong and I. S. Lee, "Comparison of Thymidine Phosphorylase Expression and Prognostic Factors in Gallbladder and Bile Duct Cancer," BMC Cancer, Vol. 10, No. 1, 2010, p. 564. doi:10.1186/1471-2407-10-564

[19] W. R. Jarnagin, D. S. Klimstra, M. Hezel, M. Gonen, Y. Fong, K. Roggin, K. Cymes, R. P. De Matteo, M. D'Angelica, L. H. Blumgart and B. Singh, "Differential Cell Cycle-Regulatory Protein Expression in Biliary Tract Adenocarcinoma: Correlation with Anatomic Site, Pathologic Variables, and Clinical Outcome," Journal of Clinical Oncology, Vol. 24, No. 7, 2006, pp. 1152-1160. doi:10.1200/JCO.2005.04.6631

[20] W. B. Kim, H. J. Han, H. J. Lee, S. S. Park, T. J. Song, H. K. Kim, S. O. Suh, Y. C. Kim and S. Y. Choi, "Expression and Clinical Significance of Cell Cycle Regulatory Proteins in Gallbladder and Extrahepatic Bile Duct Cancer," Annals of Surgical Oncology, Vol. 16, No. 1, 2009, pp. 23-34. doi:10.1245/s10434-008-0182-X 